\title{
The influence of the carbohydrate levels on viability of Stagonospora cirsii drying mycelium
}

\author{
Sofia Sokornova ${ }^{1,{ }^{*}}$, Galina Frolova ${ }^{1}$, Alexey Shavarda ${ }^{2,3}$, Natalia $_{\text {Pavlova }}{ }^{1}$ and Alexander \\ Berestetskiy $^{1}$ \\ ${ }^{1}$ All-Russian Institute of Plant Protection, 196608, St. Petersburg, Russia \\ ${ }^{2}$ Komarov Botanical Institute RAS, 197376, St. Petersburg, Russia \\ ${ }^{3}$ Saint Petersburg State University, Research park, Centre for molecular and cell technologies, \\ 199034, Russia
}

\begin{abstract}
The Stagonospora cirsii mycelium is considered as the infectious basis of a potential mycoherbicide for the control of Canadian thistle and perennial sow thistle. Successful commercialization of mycoherbicides is often constrained by poor drying survival. In this study was shown that the highest viability of mycelium S. cirsii during drying is achieved in the stationary phase of growth. The mycelium in the stationary phase is characterized by maximum level of carbohydrates. We suggest the level of arabitol as a criterion evaluation of the mycelium resistance to drying. Culturing conditions, and especially the fermentation time, allow prediction of polyols and trehalose levels, which are very critical in enhancing the storage life and efficacy of biological control agent.
\end{abstract}

\section{Introduction}

Canadian thistle (Cirsium arvense) and perennial sow thistle (Sonchus arvensis) are highly aggressive weeds of Asteraceae family. The Stagonospora cirsii mycelium is considered as the infectious basis of a potential mycoherbicide for the control of these weeds. Successful commercialization of mycoherbicides is often constrained by poor drying survival and shelf life [1]. The strain selection, the adjustment sources of carbon and nitrogen and the duration of cultivation can influence on mycelium resistance to stress factors. Earlier it was shown that in the stationary phase of growth the S. cirsii strain C163 survives drying better than in other phases [2-3]. Then we hypothesized that the age of the culture would similarly affect to other strain of $S$. cirsii. We also wanted to make sure that increasing the level of endogenous carbohydrates would improve the survival of these strains.

\section{Materials and methods}

\footnotetext{
* Corresponding author: svsokornovs@vizr.spb.ru
} 
The strains of S. cirsii C-163, C-252, C-211, C-345, 14/15 (the host plant C. arvense) and S-79, S-39, 4-10, S-47, O-15.35 (the host plant S. arvensis) were obtained from the collection of pure cultures of the Toxicology and Biotechnology Laboratory of the AllRussian Institute of Plant Protection. The strains were stored at $5^{\circ} \mathrm{C}$ on potato-glucose agar (PGA). A 10-day fungal cultures grown on PGA used as an inoculum. S. cirsii mycelium was obtained via cultivation in $250-\mathrm{mL}$ flasks with $50 \mathrm{~mL}$ of liquid nutrient medium (sucrose - $30 \mathrm{~g} / \mathrm{l}$, soybean meal - $14 \mathrm{~g} / \mathrm{l}, \mathrm{KH}_{2} \mathrm{PO}_{4}-1 \mathrm{~g} / 1, \mathrm{MgSO}_{4} \times 7 \mathrm{H}_{2} \mathrm{O}-0.5 \mathrm{~g} / \mathrm{l}$ ). Incubation was carried out at a temperature of $24^{\circ} \mathrm{C}$ on a shaker Innova 42 (Edison, NJ, USA) (180 rpm) during 3, 6 and 9 days [4]. The mycelium was separated from the culture fluid via filtration through a paper filter (Red ribbon) with a vacuum pump. The $200 \mathrm{mg}$ of mycelium (humidity $85-90 \%$ ) was ground in liquid nitrogen in a mortar with a pestle. Crude extract was obtained by ethanol $(70 \%)$ extraction at $80^{\circ} \mathrm{C}$ from this drag. The analysis of extracts was done with GC-MS. Analysis was performed by an Agilent5860 chromatograph using Agilent ChemStation E.02.02.1431 software (Agilent Technologies, US). Separation was provided with a capillary column $30 \mathrm{~m}$ long, $0.25 \mathrm{~mm}$ in diameter, and stationary phase film (95\% dimethylpolyoxane, $5 \%$ diphenyl), at a thickness of $0.1 \mu \mathrm{m}$. The following conditions was used: helium flow rate, $1 \mathrm{~mL} / \mathrm{min}-1$ and evaporator temperature, $230^{\circ} \mathrm{C}$ at a flow split ratio of 1:20. The temperature conditions of the column thermostat were the following: initial temperature of $70{ }^{\circ} \mathrm{C}$, increased by $6^{\circ} \mathrm{C} / \mathrm{min}-1$ up to $340^{\circ} \mathrm{C}$. The peaks were recorded by an Agilent 5975S mass selective detector (Agilent Technologies, US) in the total ion recording mode with a frequency of 1.8 scans per second. Electron impact ionization was performed at $70 \mathrm{~V}$ and an ion source temperature of $230^{\circ} \mathrm{C}$

Determination of retention index was performed using a calibration based on standard saturated hydrocarbons. A quantitative interpretation of the metabolite profile was carried out over the peak areas of the total ion current using the internal standard method by program UniChrom (www.unichrom.com). The amount of metabolite was determined per milligram of dry mycelium. The standard curve was defined from standards containing polyols (inositol, mannitol, arabitol, erythritol, sorbitol, etc.) and sugars (trehalose, sucrose, glucose, fructose, etc.).

\section{Results and discussion}

We confirmed that drying survival of mycelium $S$. cirsii depends on culture age (Fig. 1). In all cases, the best viability of mycelium during drying is achieved in the stationary phase of growth. In turn, the culture age significantly influenced the levels of endogenous polyols (Fig. 2). On the example of two strains $S$. cirsii, we found that increasing endogenous carbohydrates level in the mycelium is positively correlated with the survival of propagules. It is known that biochemical adaptation of fungi to temperature stress provided by carbohydrate protectors varies depending on taxonomy [5-6]. The level of trehalose in the mycelium of the strains $S$. cirsii was high, but was not significantly dependent on the cultivation time (Fig. 2). Apart from disaccharide trehalose, a number of compounds capable of protective functions under stress are presently known. They include polyols, some amino acids and other compounds [5-6]. Mannitol, sorbitol, and arabitol were predominant polyols in mycelium of strains S. cirsii. After $144 \mathrm{~h}$ cultivation, the highest concentrations of arabitol and mannitol were $3.4 \pm 0.2(2.9 \pm 0.7) \mathrm{mg} / \mathrm{g}$ dry weight and $3.6 \pm$ $0.6(2.8 \pm 0.5) \mathrm{mg} / \mathrm{g}$ for strain C-163 and strain 4-10, respectively. Endogenous arabitol and mannitol has recently been shown to improve shelf life of Metarhizium brunneum [7]. It is important to note that not much is known about the physiological role of arabitol [8]. We suggest the level of arabitol as a criterion for a prediction of the mycelium resistance to 
drying. Culturing conditions, and especially the fermentation time, allow prediction of polyols and trehalose levels, which are very critical in enhancing the storage life and efficacy of biological control agent.

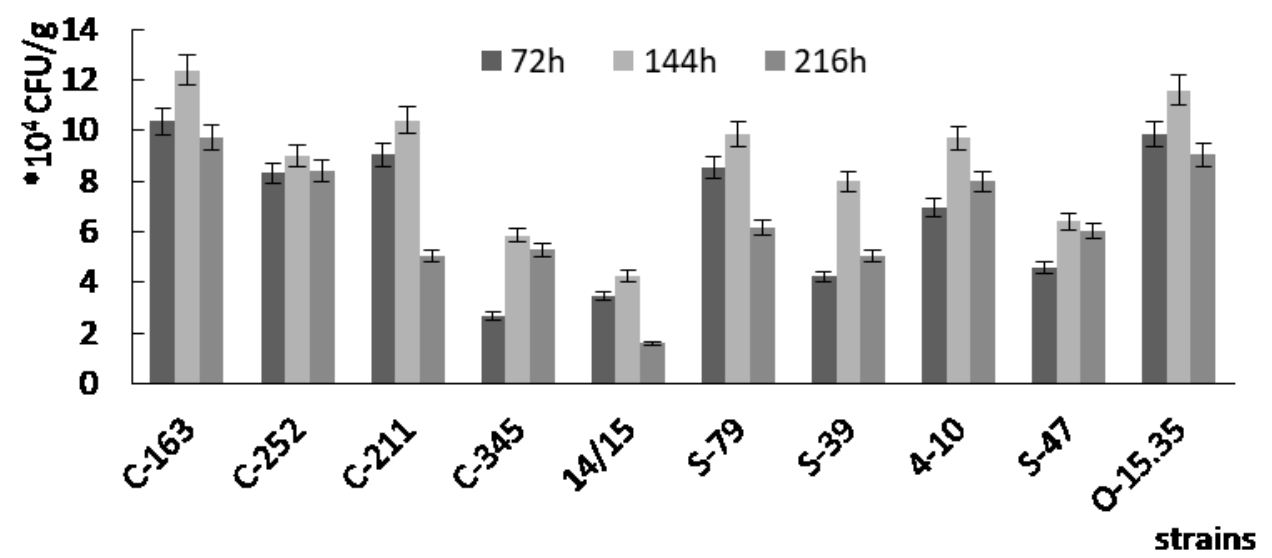

Fig. 1 Influence of drying on the viability $S$. cirsii mycelium. $\mathrm{LCD}_{0.05}=0.8 * 10^{4}(\mathrm{CFU} / \mathrm{g})$

A

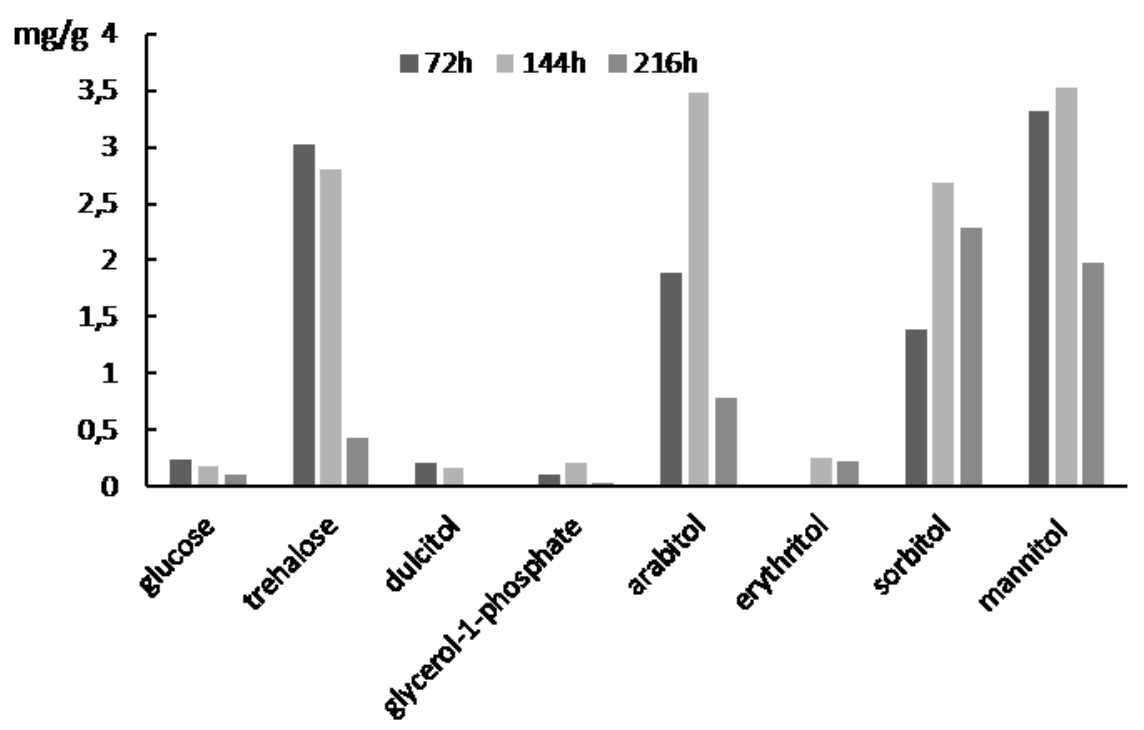


B

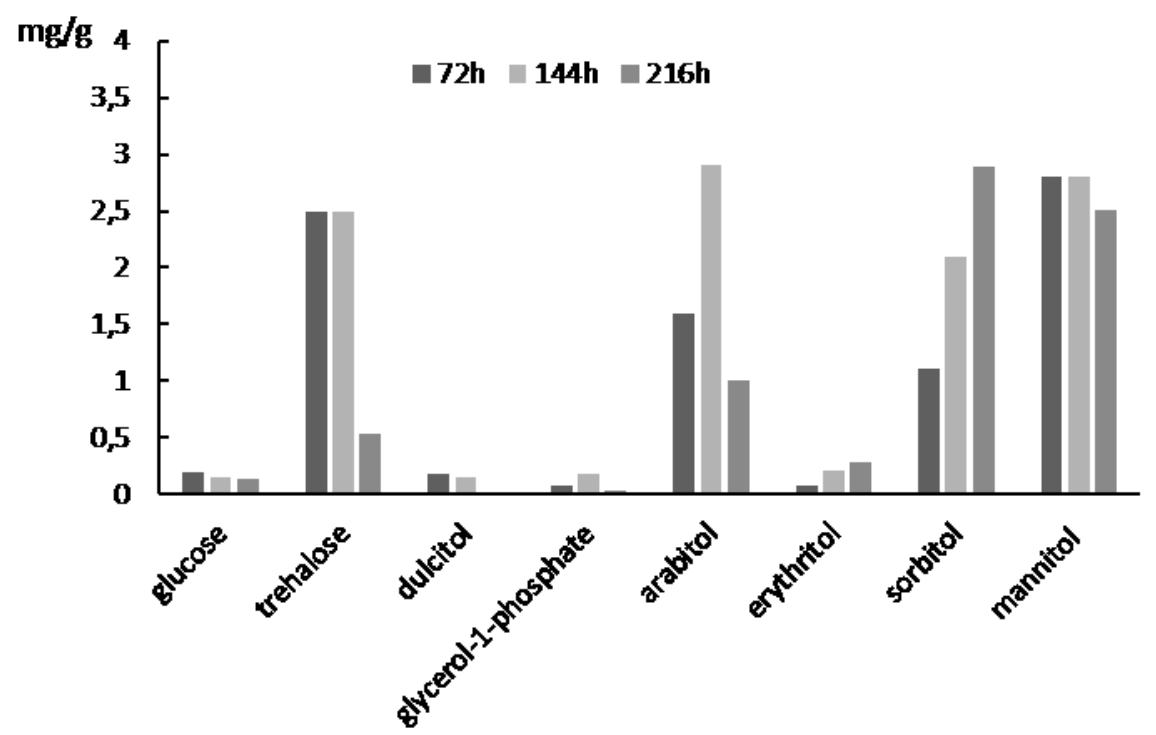

Fig. 2. Influence of culture age on the accumulation of endogenous carbohydrates in S. cirsii mycelium A - strain C-163, B - strain 4-10. $\mathrm{LSD}_{0.05}=0.03$ (mg/g dry weight)

This work was supported by RSF grant 16-16-00085.

\section{References}

1. K. Bailey, S. Boyetchko, T. Langle. Biological control : theory and application in pest management, 52, 3, 221-229 (2010). ISSN 1049- 9644

2. S.V. Sokornova, A.O. Berestetskiy. Sel'skokhozyaistvennaya Biologiya [Agricultural

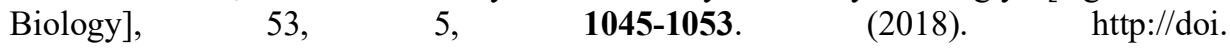
org/10.15389/agrobiology.2018.5.1054eng

3. N.A. Pavlova, S.V. Sokornova, A.O. Berestetskiy. Plant Protection News, 4, 51-53 (2017). http://vestnik.vizrspb.ru/ru/assets/documents/issues/2017/4/vestnik_17-48_Pavlova.pdf

4. N.A. Pavlova, S.V. Sokornova. Plant Protection News, 4, 67-69 (2018). http: //doi. org/10.31993/2308-6459-2018-4(98)-67-69

5. Feofilova, E.P., Tereshina, V.M., and Gornova, I.B., Microbiology (Moscow), 63, 5, 442-445 (1994).

6. E.P. Feofilova, A.I. Usov, I.S. Mysyakina, G.A. Kochkina. Microbiology (Moscow), 83, 184-194 (2014).

7. V. Krell, D. Jakobs-Schoenwandt, M. Persicke, A.V. Patel. World J. Microbiol. Biotechnol. 34, 8, 108 (2018).

8. S. Meijer, G. Panagiotou, L. Olsson, J. Nielsen. Biotechnol. Bioeng. 98, 462-475 (2007) 